\title{
K-Dominance and Size effect in Mode I Fracture of Brittle Materials with Low to Medium Porosity
}

\author{
Dimitra Touliatou, Marcus A. Wheel ${ }^{(*)}$ \\ Department of Mechanical and Aerospace Engineering \\ University of Strathclyde \\ Glasgow, G1 1XJ, United Kingdom \\ ${ }^{\left({ }^{*}\right)}$ Corresponding author, e-mail: marcus.wheel@strath.ac.uk
}

\begin{abstract}
Linear Elastic Fracture Mechanics usually only considers the singular stresses when describing the conditions under which fracture would occur in a brittle material. However, it is becoming more widely recognised that non-singular stresses can become significant depending on the geometry and configuration of the specimen. This study investigates the impact of non-singular stresses on the stress intensity of low to medium porosity brittle materials. To address this, discrete finite element models of Double Cantilever Beam (DCB) samples were created and the full near-tip stress field in mode I loading was numerically evaluated. A parametric study was conducted, examining the influence of overall specimen size, material porosity and crack tip location relative to the nearest void. Results indicate a prominent size effect on the stress intensity at the crack tip of porous materials, with smaller specimen exhibiting tougher behaviour than their respective larger counterparts. This size effect, which is amplified with increasing porosity, is closely correlated with the variation of non-singular stresses, both parallel and normal to the crack plane. A model to predict the behaviour of porous specimen for different sizes is suggested based on the findings.
\end{abstract}

Keywords: Porous materials; Non-singular stresses; Size effect; Brittle fracture

\section{Introduction}

Porous materials offer a plethora of useful attributes including unique specific properties, low density and high thermal and acoustic insulation. These properties make them attractive to various applications within the automotive, aerospace, aeronautical and ship building industries $[1,2]$ where they are mainly utilised as cores of composite sandwich structures. In the fields of geoscience and geoengineering, the understanding of underground stress fields around single fractures and fracture networks in porous media are of outmost importance for the investigation of ways to achieve more efficient geothermal energy harvesting, water resource conservation, $\mathrm{CO}_{2}$ sequestration, and ensuring safe resource exploitation in the oil and gas industry amongst other matters $[3,4]$.

Despite their growing popularity, the behaviour of porous materials is not yet fully understood and quantified. In fact, there currently exists no standardized testing procedure for the fracture of brittle porous materials, while a number of studies have used testing standards appropriate 
to non-porous polymers which assume that Linear Elastic Fracture Mechanics (LEFM) is applicable[5-7].

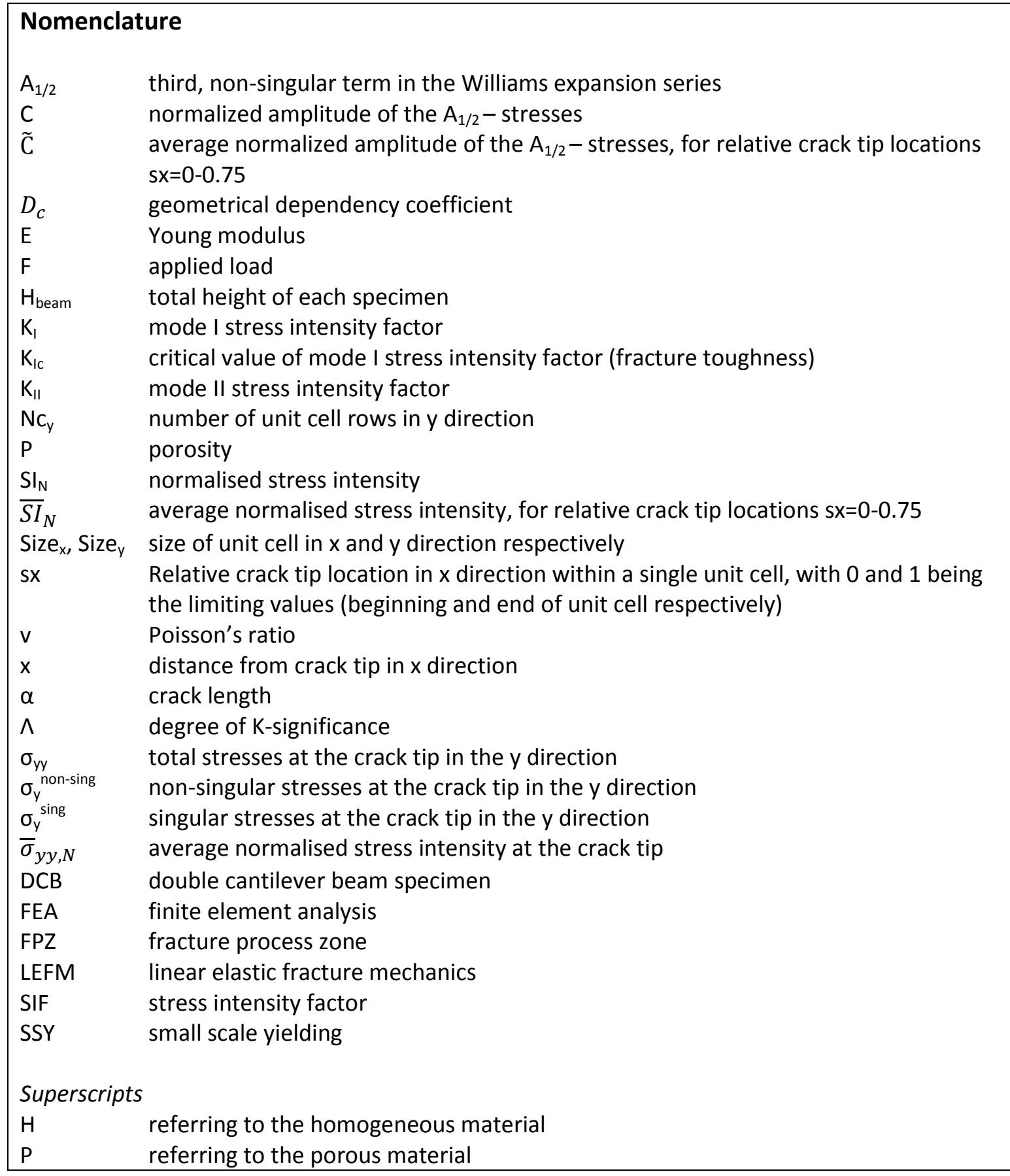

LEFM is predicated on the notion of Small Scale Yielding (SSY) which assumes that the Fracture Process Zone (FPZ) is small compared to other relevant dimensions of the specimen. In turn, the stress intensity factor (SIF) is assumed to be a characteristic constant for a given material, loading and specimen geometry, capable of fully describing the stress field around the crack tip 
$[8,9]$. This assumption, however, is limited and can often produce unrealistic results. For example, even for the same material, specimens of different configurations can exhibit apparently different fracture toughness values which can be more accurately predicted by taking into account additional crack tip parameters [10-16].

In heterogeneous materials, local discontinuities in the matrix material can influence the stress field in the vicinity of the heterogeneity, leading to the appearance of local SIFs at different locations with respect to the void [17-19]. Han and Chen [17], while studying the influence of a microvoid on the SIF of a brittle material, attributed its oscillation to the relaxation of T-stress, a constant stress parallel to the crack, which occurs near the void and results in either an amplification or a shielding effect. These studies refer to individual pores in infinite media, which implies that the size of the heterogeneity is insignificant compared to the total size of the specimen.

However, when the size of the heterogeneity becomes comparable to that of the specimen, heterogeneous materials have been observed to exhibit crack tip stresses contradicting the usual size scaling predicted by LEFM. These size effects can cause severe discrepancies and unexpected fracture events if they are not taken into account. For quasi-brittle heterogeneous materials such as polymer foams [5-7,20], composites [21,22], and cement [23-25], this behaviour is normally attributed to the material's transition to plasticity in very small scales.

Wheel et al. [26-29] have investigated this size effect in materials with periodic porosity subject to bending loads and have thereby described their behaviour in the context of micropolar media. Their studies showed that when the size of the pores remains negligible to the total dimensions of the specimen its flexural stiffness converges to the homogeneous case. However, when the specimen gets smaller while remaining geometrically similar, there is significant stiffening.

This study extends these findings to the fracture of low to medium porosity materials of values ranging from $5-45 \%$. Key parameters including porosity, specimen size and crack location with respect to the voids have been considered and their effect on the stress intensity at the crack tip and K-dominant zone size within these materials examined. We investigate the full near-tip stress field and estimate both the singular and non-singular stresses for specified loads that stress the crack-tip below the critical stress intensity factor. The adopted approach focuses on the local stress field around the crack tip, but does not include any assumptions regarding softening, as adopted in quasi-brittle materials. Crack propagation is not considered within the scope of this paper.

\section{Methodology}

\section{1. $\quad$ Finite Element Model}

Two-dimensional plane strain models of Double Cantilever Beam (DCB) specimens were created using the commercial finite element analysis (FEA) software ANSYS. Each model was created by repeatedly regenerating a rectangular unit cell with a centrally located circular void, shown in 
Figure $1 \mathrm{~d}$, across the length and depth of the specimen, the geometry of which can be seen in Figure 1a. The dimensions of the unit cell in the $x$ and $y$ direction are Size $_{x}=3.5 \mathrm{~mm}$ and Size $_{\mathrm{y}}=\sqrt{ } 3 \mathrm{Size}_{\mathrm{x}} / 2$ respectively. This configuration is such that the voids form an equilateral triangular arrangement (Figure 1c), implying approximately isotropic behaviour of the macroscopic material [27]. The porosity of the specimen is varied from approximately 5 - $45 \%$ by changing the void diameter as detailed in Table 1. A homogeneous model was created for reference and validation using the same mesh configuration and material properties as used for the porous models.

\begin{tabular}{|c|c|c|c|c|c|c|}
\hline Void radius $(\mathrm{mm})$ & 0.82 & 1.16 & 1.64 & 2 & 2.32 & 2.46 \\
\hline Sample porosity $\mathrm{P}(\%)$ & 4.98 & 9.96 & 19.96 & 29.61 & 39.85 & 44.80 \\
\hline
\end{tabular}

Table 1. Specimen porosity for each given void radius.

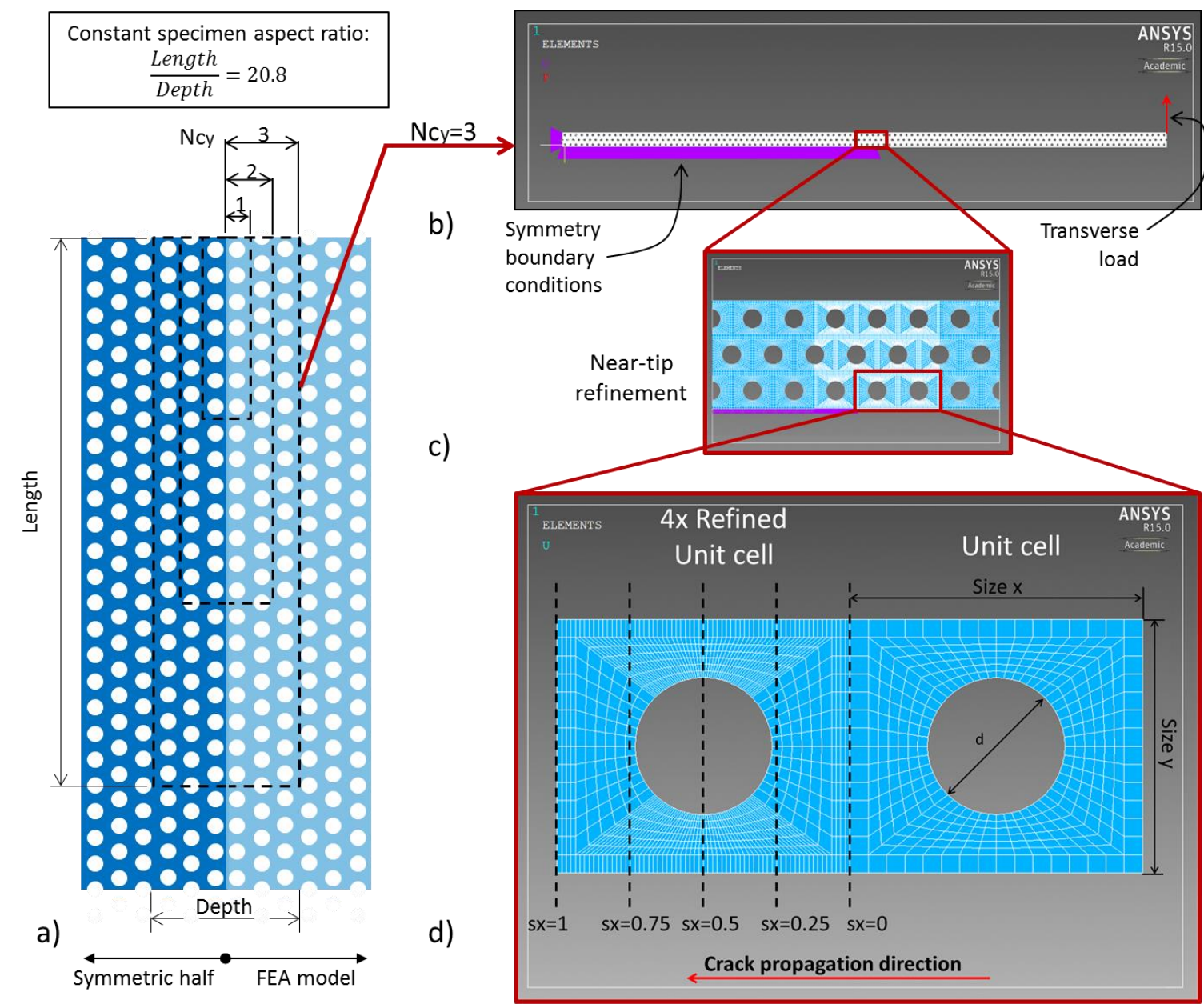

Figure 1. a) Illustration of the different specimen sizes (dashed lines) cut from an infinite sheet of material, b) Boundary conditions and loading for a model with $\mathrm{Ncy}=3$, c) Near-tip refined mesh for a model with $\mathrm{Ncy}=3$, d) Refined and non-refined unit cell. The crack tip locations relative to the void sx are depicted on the image. The crack is assumed to propagate with a direction from sx=0 to sx=1. 
Each unit cell is paved with a structured mesh, using element Plane 183, an 8-node twodimensional structural element. The boundaries of each unit cell have been meshed with equal sized elements by 14 and 16 elements in the horizontal, $x$, and vertical, $y$, directions respectively (right cell of Figure 1d). Near the crack tip, the horizontal boundaries of the immediately adjacent cells are refined $4 x$ times, giving a total of 56 and 16 elements in the horizontal and vertical direction respectively (left cell of Figure $1 \mathrm{~d}$ ). Five different locations relative to the nearest void have been considered for the crack tip position, starting at the edge of the unit cell $(s x=0)$ and advancing the crack tip by one quarter of its length up to the other end of the unit cell $(s x=1)$ (Figure $1 d)$.

A mesh sensitivity study was conducted (Figure 2) to assess numerical uncertainties especially in regions where singularities appear. In order to keep the aspect ratio of the elements within the acceptable limits, large changes in the refinement of the near-tip area in the horizontal direction mandated proportional changes in the refinement of the far-field mesh which controls the $y$ direction mesh distribution (see Figure 1d). Below an element size of just above $0.05 \mathrm{~mm}$, the solution seems to converge with minimal fluctuations. At that level of refinement, two models with different far-field refinements were run to examine the impact of the element's aspect ratio on the FEA solution. Both the refined and non-refined far-field mesh gave almost identical results. Thus, the non-refined far-field mesh with $0.05 \mathrm{~mm}$ near-tip element size, as described in the previous paragraph, was selected for the simulations.

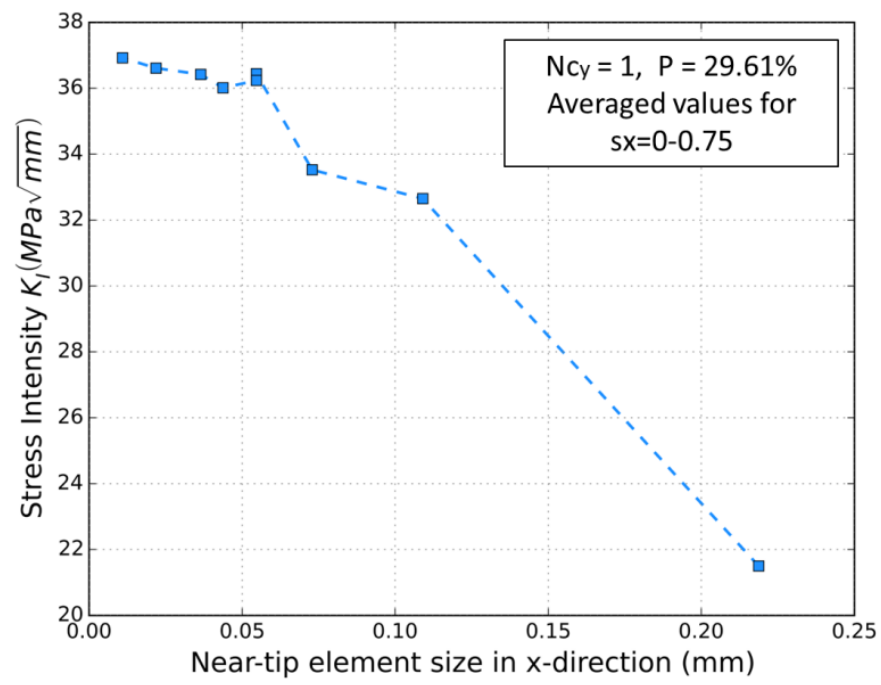

Figure 2. Mesh convergence study. At an element size just above $0.05 \mathrm{~mm}$ two overlapping points are seen which represent two different levels of far-field refinement. The non-refined far-field mesh with a near-tip element size of approximately $0.05 \mathrm{~mm}$ was selected for the simulations presented below.

Geometrically similar models of six sizes were created for each case, containing from 1 to 6 rows of unit cells (number of unit cells in y-direction $\mathrm{Nc}_{\mathrm{y}}$ ) arranged symmetrically about the crack (Figure 1a). Importantly, geometrical similarity of the specimen was maintained by keeping the specimen aspect ratio constant to 20.8 (Figure 1a). In order to reduce computational expense and due to the symmetrical nature of a Mode I test only half of the model was generated (Figure 1b). It should be noted that due to the symmetric nature of the model, the porosity around the 
crack is symmetric and does not preserve the triangular pattern used in the rest of the beam (Figure 1c). The crack length $\alpha$ is similarly scaled and is approximately $\alpha=10 H_{\text {beam }}$, where $\mathrm{H}_{\text {beam }}$ is the total height of the symmetric beam.

A transverse tensile point load is applied at the free edge of the specimen (Figure 1b). The load is varied so that the stress at the crack tip remains constant for all specimen sizes and crack lengths in the homogeneous case. All models are considered different structural configurations of the same, perfectly brittle material and thus a matrix Young's modulus of E=3 GPa and Poisson ratio $\mathrm{v}=0.35$ is used for all models.

\subsection{Analysis of Numerical Results}

The near-tip stress field of a sharp crack in a perfectly brittle material in Mode I loading can be described with William's asymptotic expansion [30]. Considering a polar coordinate system $(r, \theta)$ with its origin at the crack tip [31] the stress components are given by:

$$
\left[\begin{array}{c}
\sigma_{r r} \\
\sigma_{\theta \theta} \\
\sigma_{r \theta}
\end{array}\right]=\frac{K_{I}}{\sqrt{2 \pi r}} \frac{1}{4}\left[\begin{array}{c}
-\cos \frac{3 \theta}{2}+5 \cos \frac{\theta}{2} \\
\cos \frac{3 \theta}{2}+3 \cos \frac{\theta}{2} \\
\sin \frac{\theta}{2}+\sin \frac{3 \theta}{2}
\end{array}\right]+4 A_{0}\left[\begin{array}{c}
\cos ^{2} \theta \\
\sin ^{2} \theta \\
-\sin \theta \cos \theta
\end{array}\right]+\frac{3 A_{1 / 2} r^{1 / 2}}{4}\left[\begin{array}{c}
3 \cos \frac{\theta}{2}+\cos \frac{5 \theta}{2} \\
\cos \frac{\theta}{2}-\cos \frac{5 \theta}{2} \\
\sin \frac{\theta}{2}-\sin \frac{5 \theta}{2}
\end{array}\right]+\begin{gathered}
\text { Higher } \text { Order (1) } \\
\text { Terms }
\end{gathered}
$$

The first term of (1) is singular and is described by the SIF, $\mathrm{K}_{1}$. In LEFM, the SIF is a parameter that on reaching a critical value, $\mathrm{K}_{\mathrm{I}}$, a unique material property termed the fracture toughness, can be used to accurately describe the fracture initiation process in the material. The second and third terms are the $T$-stress (where $T=4 A_{0}$ ) and the non-singular $A_{1 / 2}$-stress, respectively. These terms depend on the geometry and finite boundaries near the crack tip. Further higher order terms are not considered in this study.

On approaching the crack tip $(r=0)$, the first term tends to infinity while the higher order terms remain constant or tend to zero. Thus, there exists a region in which the fracture process is governed almost exclusively by the singular stress and is identified as the K-dominant zone. In LEFM it is a fundamental assumption that this singularity dominated zone is sufficiently large to imply that the SIF alone fully describes the stress field around the crack tip. [9]

Indeed, it has been demonstrated previously that when the size of the K-dominant zone is large enough to engulf the fracture process zone completely, then this assumption is valid and the estimated stress at the crack tip is independent of the specimen's configuration. However, when that requirement is not met and higher order terms become significant, the fracture toughness is apparently no longer constant. While the non-singular stresses may not have a direct contribution on the value of $K_{1}$, they can indirectly influence its magnitude. [13]

In order to estimate $K_{1}$ from the numerical results we used the projection method as presented in [32] and briefly described below. Considering the $y$-direction normal stress distribution $\left(\sigma_{\theta \theta}\right)$ ahead of the crack tip $(\theta=0)$ only, equation (1) can be transformed as: 


$$
\sigma_{y y}(x)=\frac{K_{I}}{\sqrt{2 \pi x}}+3 A_{1 / 2} \sqrt{x} \quad \Rightarrow \quad \sigma_{y y}(x) \sqrt{2 \pi x}=K_{I}+\left(3 \sqrt{2 \pi} A_{1 / 2}\right) x
$$

where $x$ is equivalent to $r$ in $(r, \theta)$ when $\theta=0$.

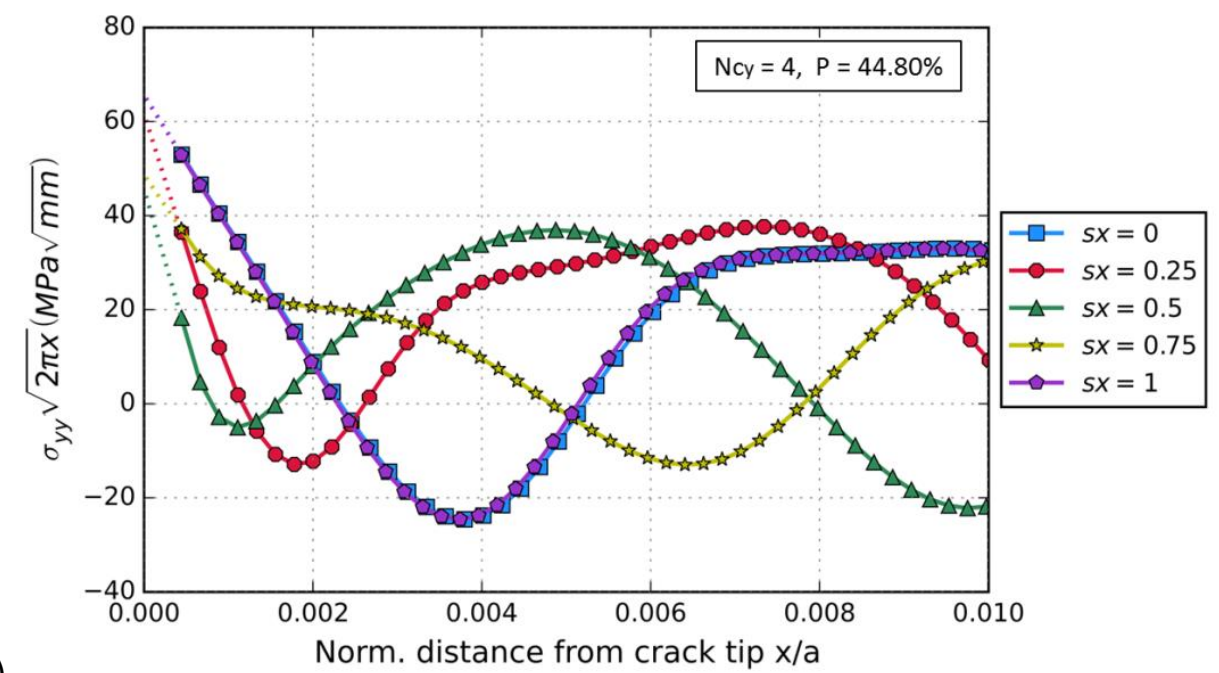

a)

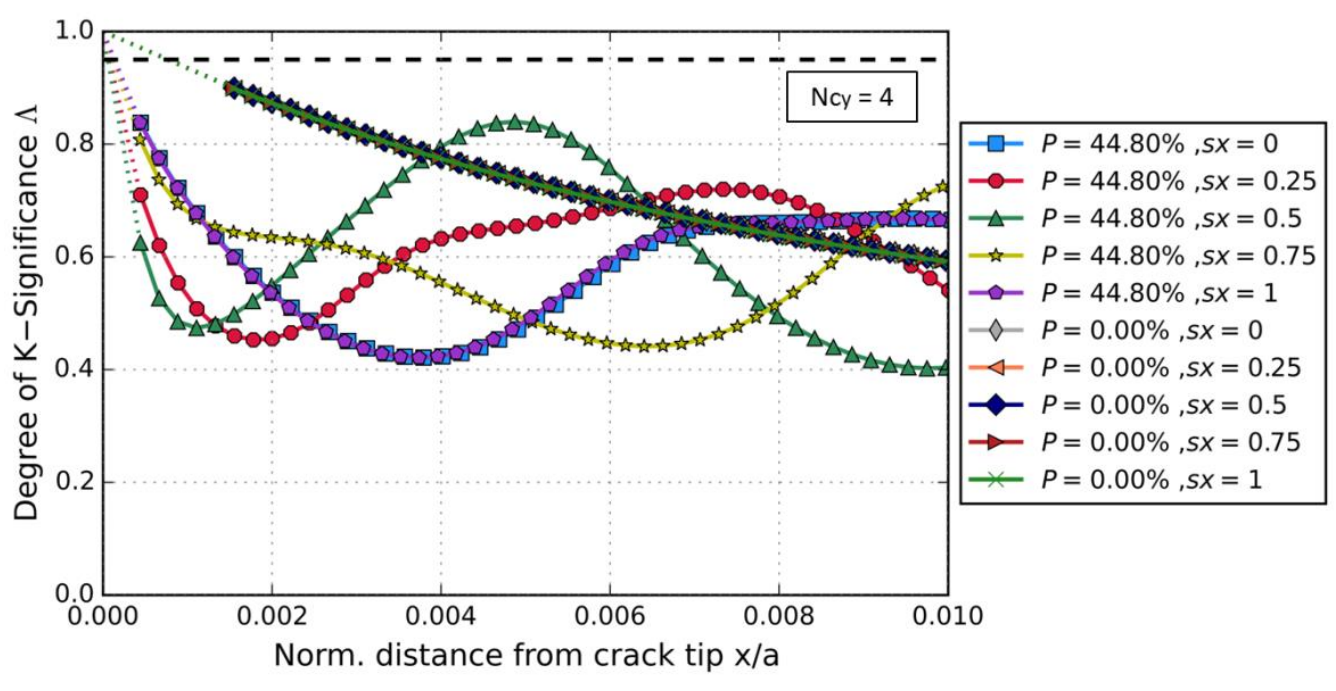

Figure 3. a) Projection method to estimate the magnitude of the stress intensity at the crack tip (SIF), b) Linear extrapolation of the degree of K-significance $\Lambda$ to estimate the size of the K-dominant zone. The dashed line represents $\Lambda=95 \%$.

In Figure 3a, the stress intensity variation is plotted against the distance ahead of the crack tip, normalized by the crack length for different crack tip positions in specimens comprised of four rows of unit cells and a constant porosity of $44.8 \%$. For homogeneous materials this variation is linear with an equal gradient at all size scales. In this study, the gradient of the variation is referred to as the normalized amplitude of $A_{1 / 2}$-stresses and can be quantified as $\mathrm{C}=$ $\left(3 \sqrt{2 \pi} A_{1} / 2\right)$ a. To estimate $C$ for the porous materials investigated, only the linear part of the variation, immediately ahead of the crack tip, is considered. Due to numerical limitations in accurately calculating stresses in the vicinity of the crack tip singularity, a straight line is fitted 
onto the linear part of each stress variation and extrapolated back to zero radius (i.e. dotted lines in Figure 3a).

At infinitesimal distances from the crack tip, non-singular terms become negligible compared to the first term of equation (2), which in turn takes the form:

$$
\lim _{x \rightarrow 0} \sqrt{2 \pi x} \sigma_{y y}(x)=K_{I}
$$

The values of the stress intensity estimated by this method are in good agreement with those estimated from ANSYS directly using the Interaction Integral Formulation, while, for the homogeneous case, the value estimated with the projection method has a closer correlation to the analytical estimation of the apparent stress intensity for DCB specimen (Table 2).

\begin{tabular}{|c|c|c|c|}
\hline & Analytical & $\begin{array}{c}\text { Interaction } \\
\text { Integral }\end{array}$ & $\begin{array}{c}\text { Projection } \\
\text { method }\end{array}$ \\
\hline $\begin{array}{c}\text { Apparent stress } \\
\text { intensity } \\
\mathbf{K}_{\mathbf{I}}(\mathbf{M P a} \sqrt{\mathbf{m m})}\end{array}$ & 31.84 & 32.86 & 31.81 \\
\hline $\begin{array}{c}\text { Discrepancy from } \\
\text { analytical }\end{array}$ & - & $3.15 \%$ & $0.09 \%$ \\
\hline
\end{tabular}

Table 2. Comparison of $K_{1}$ values of the homogeneous case estimated analytically, using the interaction integral formulation in ANSYS and using the projection method described in [32].

It can be seen in Figure 3a that the extrapolated stress at the crack tip used for the calculation of the stress intensity depends on the slope of each curve, and is therefore indirectly affected by the near-tip stress distribution. While the $A_{1 / 2}$-stresses are negligible right at the crack tip, they may nonetheless be significant in the near-tip region, thereby indirectly affecting the value of the apparent stress intensity.

In order to normalise the results, the $K_{1}$ estimated by extrapolation for the porous materials was normalized with respect to the $K_{1}$ of the homogenous case, which is a constant value $\left(K_{I}{ }^{H}=\right.$ $31.81(\mathrm{MPa} \sqrt{\mathrm{mm}})$. From the definition of equation (3) on $\mathrm{x}$ approaching zero, this normalising is equivalent to the ratio of the opening stress at the crack tip in the porous material to the opening stress at the crack tip in the homogeneous material, henceforth referred to here as the normalised stress intensity $\mathrm{SI}_{\mathrm{N}}$, where:

$$
\mathrm{SI}_{N}=\frac{K_{I}^{P}}{K_{I}{ }^{H}}=\frac{\sigma_{y y}{ }^{P}}{\sigma_{y y}{ }^{H}}
$$

It is important to remember that the values of stress intensity and opening stress refer to models with loading scaled in such a way that they remain constant for all specimen sizes with zero porosity. Thus, it merely reflects the magnitude of the opening stress at the crack tip for a given load and not the critical value of stress that the material could actually withstand before initiating the crack $\left(K_{1}\right)$, which is not considered in this study.

The K-dominant region was considered as the zone around the crack tip where $\mathrm{K}_{1}$ contributes to more than $95 \%$ of the opening stress at the crack tip (see horizontal dashed line in Figure $3 \mathrm{~b}$ ). The degree of K-dominance $[13,15,33]$ is therefore estimated as: 


$$
\Lambda=\frac{\sigma_{y}^{\operatorname{sing}}}{\sigma_{y}^{\operatorname{sing}}+\left|\sigma_{y}{ }^{\text {non-sing }}\right|}
$$

, where

$$
\sigma_{y}^{\operatorname{sing}}(x)=\frac{K_{I}}{\sqrt{2 \pi x}} \quad(6) \quad \text { and } \quad \sigma_{y}{ }^{n o n-\operatorname{sing}}(x)=\sigma_{y y}(x)-\sigma_{y}{ }^{\operatorname{sing}}(x)
$$

\section{Results and Discussion}

In the results presented below, it is important to note that even though the specific values of stresses are influenced by the applied load, the value of the degree of K-dominance, $\Lambda$, and the size of the K-dominant zone are uniquely controlled by the specimen's geometry and the specifications of its internal mesostructure, independently of the material and load. The normalized amplitude of $A_{1 / 2}$-stresses $C$ is affected by variations in the load $F$ in a similar manner to stresses. Thus, the ratio $C / K_{1}$ remains unaffected by externally applied variations and only depends on the material's geometrical features. That makes the absolute values of the applied load non-significant in the interpretation of the results presented below.

Due to the scaling of the load to reflect changes in size, all different sizes are considered nondimensional in the sense that their absolute size is not as significant as the configuration (i.e. number of rows) they represent. This can be validated by the fact that for the homogeneous case, all different sizes would give identical values of stress intensity. In other words, when shrinking the unit cell to one quarter of the size described in the paper and creating a specimen with two numbers of rows, (whose size in absolute values would be identical to the specimens with the original unit cell size described in the paper with an $\mathrm{Ncy}=1$ ), the resulting stress intensity, both mesoscopically and macroscopically, would be identical to that of the specimen with the $4 \mathrm{x}$ larger unit cell and $\mathrm{Ncy}=2$, regardless of their difference in absolute size. Thus, it seems that the number of voids in the near-tip area influences the stress intensity at the crack tip and cannot be omitted by homogenisation of the materials geometry.

The significance of the non-singular stresses on the value of $K_{1}$ of a porous material can be identified from the FEA results. Figure 4 shows the discrepancy between the FEA solution for the stress intensity ahead of the crack tip and the analytical K-field solution which only considers the singular stress term in estimating the stress field. In the homogenous case, singular stresses seem to be the significant term in fracture, with the analytically estimated stress field being almost in total agreement with the FEA solution. However, for porous materials, considering the singular stresses alone gives an inaccurate analytical prediction of the near-tip stress field.

In the following sections, the influence of the non-singular stresses on the results derived will be presented at two focus levels: a) The porous structure level, referred to here as the meso-scale, and b) The homogenized material level, referred to as the macro-scale. 


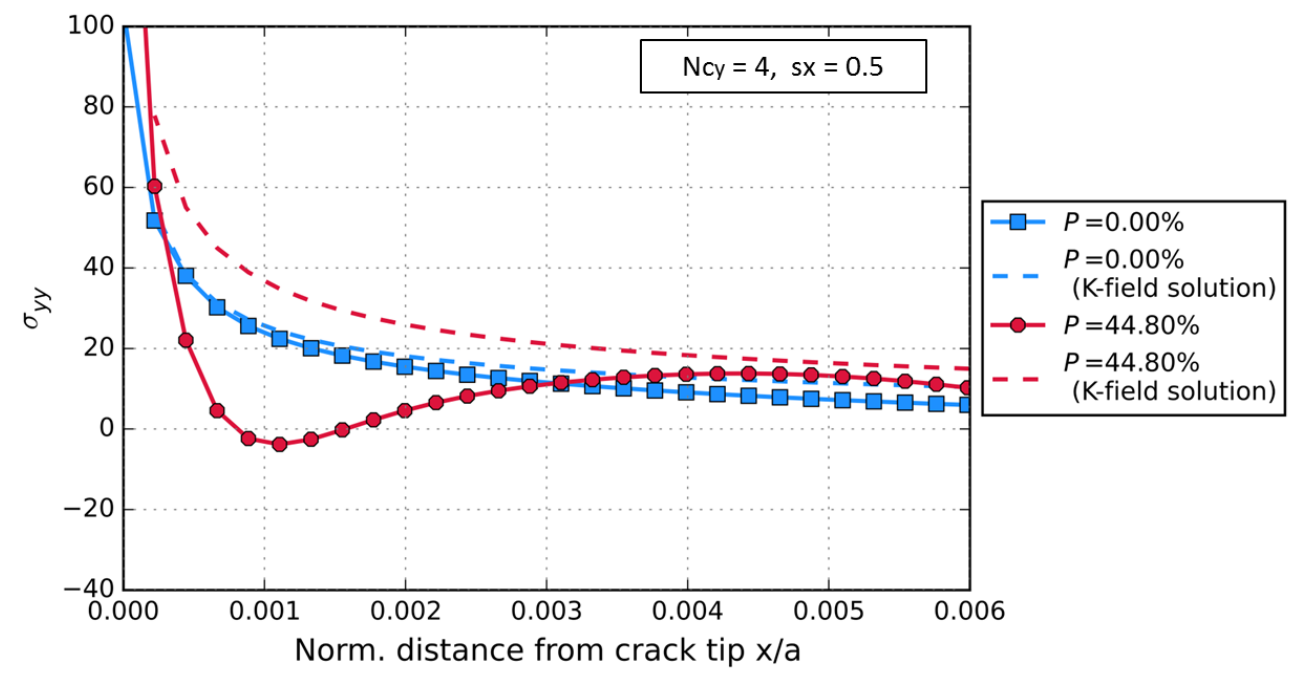

Figure 4. FEA solution (solid lines) vs analytically determined K-field solution based solely on the influence of the SIF (dashed lines).

\subsection{Mesoscale: Porous structure}

Porous materials exhibit local $K_{1}$ values which are controlled by the material's mesostructure directly in the vicinity of the crack tip. In this study, FEA results show that for all specimens the normalised stress intensity $\mathrm{SI}_{\mathrm{N}}$ follows an oscillating pattern for different crack tip locations relative to the void, which is periodically repeated for consecutive cells (Figure $5 a, b$ ). This periodicity is linked to the nature of the prescribed mesotructure, which is non-random, with voids following a regular pattern, this being symmetric about the crack plane.

It is found that the amplitude of the oscillation of $\mathrm{SI}_{\mathrm{N}}$, diminishes when the size of the heterogeneity in the near tip field becomes less significant compared to the specimen's dimensions; at both fixed specimen size (Figure 5a) (also seen in [18]) and fixed void size (Figure $5 b)$. Interestingly, the regions around the void, where the T-effect leads to either amplification or shielding, appear to invert as the size of the specimen decreases (Figure 5b). Specifically, for progressively smaller specimens the stress variation inverts, reaching a maximum at the edges of the cell (i.e. $s x=0$ and $s x=1$ ) and a minimum exactly under the void ( $s x=0.5)$. However, as the size of the specimen increases, the locations of the maxima and minima of the oscillation are repositioned to locations before $(s x=0.25)$ and after $(s x=0.75)$ the void respectively.

This oscillatory behaviour is consistent with that found during previous investigations on the variance of local SIFs at various distances and angles around a single circular void $[17,18]$ nearby the crack tip in infinite media. Any discrepancies with the fluctuations found here are attributed to the existence of neighbouring voids which further alter the stress distribution. Han et al. [17] attributed these fluctuations to the T-effect, suggesting that the x-direction non-singular Tstress is relaxed inside the voids, consequently having a direct impact on the SIF of the material. They suggested that for mode I loading, a combination of the relative location of the crack tip with the T-stress sign can either amplify or shield the material's SIF. 


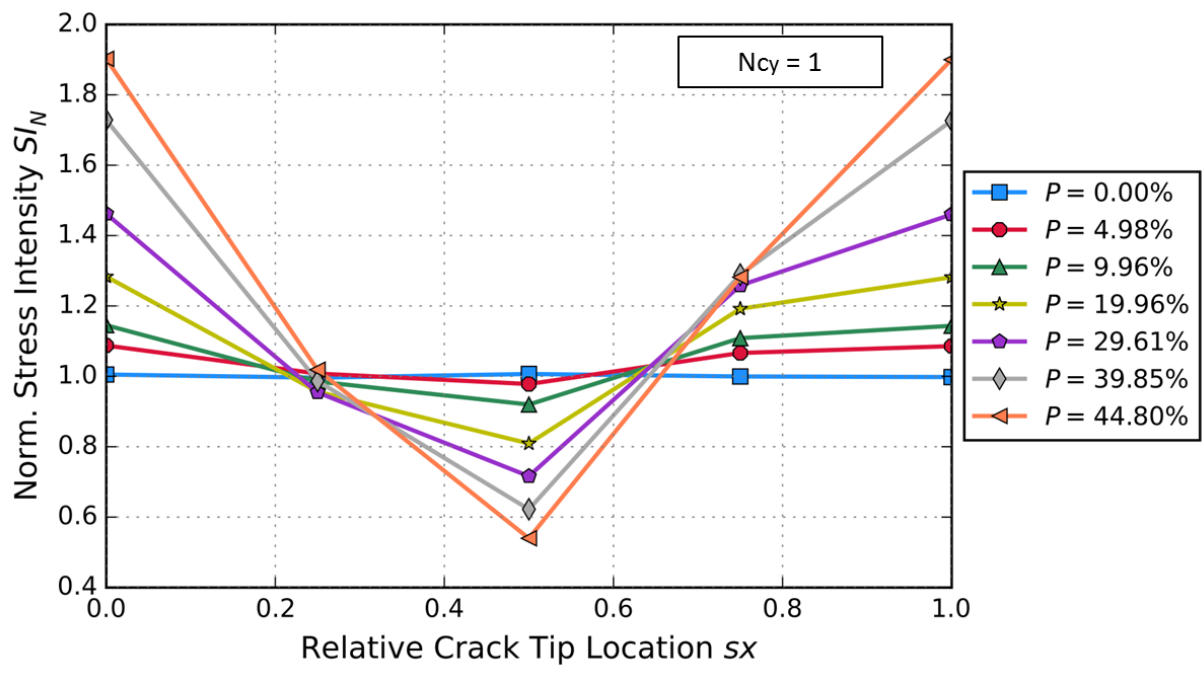

a)

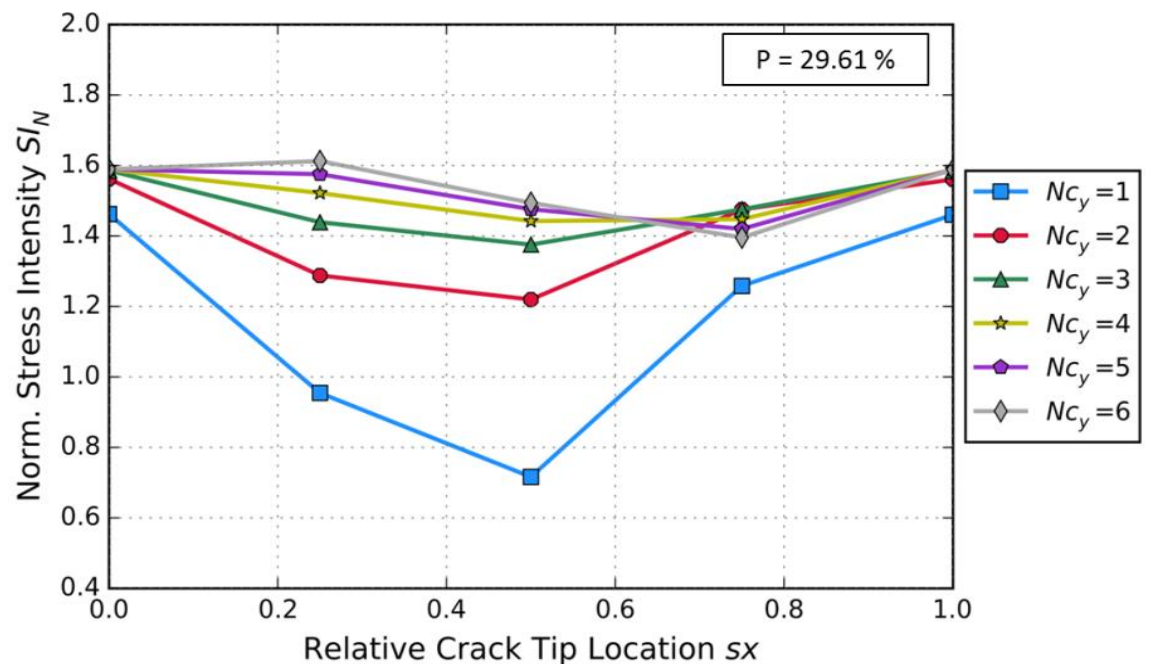

b)

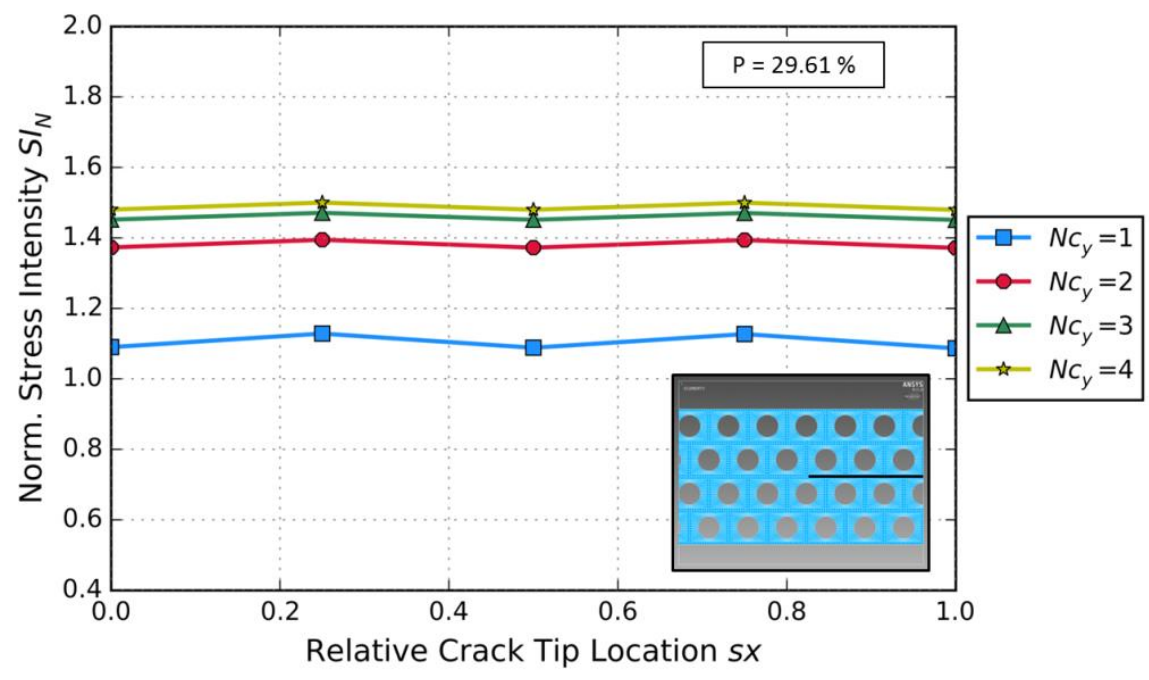

Figure 5. Fluctuations of $\mathrm{SI}_{\mathrm{N}}$ for different crack initiation locations relative to the void a) Size $\mathrm{Ncy}=\mathbf{1}$ for porosities=5$45 \%$, b) Sizes Ncy=1-6 for $30 \%$ porosity, c) Sizes Ncy=1-6 for $30 \%$ porosity model with triangularly arranged voids equilaterally to the crack. 
For comparison, a full specimen model was created with a triangular but non-symmetric void pattern adjacent to the crack plane (see inset of Figure $5 \mathrm{c}$ ), consistent with the pattern throughout each beam. The model was created as described in section 2 , with $30 \%$ porosity and was run for 1-4 rows of unit cells. The asymmetry of the voids around the crack tip introduces a mode II component in the near-tip stress field. However, the magnitude of the mode II component contributes to only approximately $7.5 \%$ of the effective mixed mode stress intensity $\left(\mathrm{K}_{\mathrm{I}}=38.53 \mathrm{MPaVmm}\right.$ and $\mathrm{K}_{\|}=2.90 \mathrm{MPaVmm}$ for $\mathrm{sx}=0.75$ where their percentile difference is the smallest, effective mixed mode stress intensity is estimated as $K_{e f f}=\sqrt{{K_{I}}^{2}+{K_{I I}}^{2}}$ ) for the smallest specimen, where the contribution of Mode II stresses is most apparent since the void size dimensions is significant compared to the specimens dimensions. Thus, predominantly mode I behaviour is assumed. The local stress intensity of these models exhibits significantly diminished oscillation throughout the unit cell, as seen in Figure $5 \mathrm{c}$. This is attributed to the averaging of the two almost contrasting, overlapping stress fields on either side of the crack plane, caused by the non-symmetric equidistant voids near the crack-tip.

It is apparent that the T-stresses are immediately influenced by the mesostructure of the material in the near-tip field and can lead to large discrepancies in the local stresses around the crack tip depending on its relative location. Apart from the amplitude of the oscillation, the mean value about which $\mathrm{SI}_{\mathrm{N}}$ oscillates increases with size, appearing to converge to a plateau for large specimens. In order to examine the extent of the effect of the T-stresses on the stress intensity at the crack tip, we focus at the neutral points, where the T-effect is zero and the stress intensity of each case is aligned with the reference homogeneous case. Each model has two neutral points, located slightly differently for each model, about the midpoint of the unit cell, in close proximity to the void.

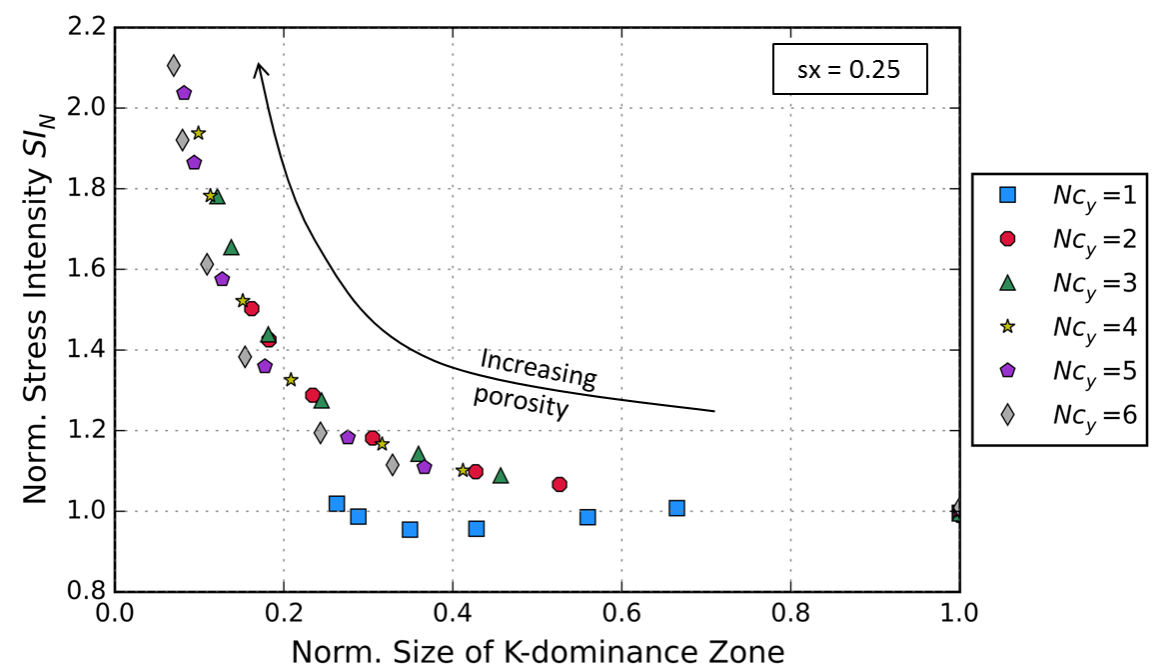

Figure 6. Variation of $\mathrm{SI}_{\mathrm{N}}$ with size of $\mathrm{K}$-dominant zone, plotted for $\mathrm{sx}=0.25$ where the effect of the T-stresses is close to zero for all cases. In all graphs porosity increases from the right to the left side of the graph.

Concentrating on the neutral point in front of the void, we use the relative crack location sx $=0.25$ as an average value for all models to plot the size of the K-dominant zone, normalised 
with the respective value of the homogenous case for each specimen size. The normalised stress intensity, $\mathrm{SI}_{\mathrm{N}}$, is plotted against this value for each model (Figure 6).We found that the variation of the significance of the $A_{1 / 2}$-stresses of 1-row specimen seems to have almost no effect on their fracture behaviour, indicating T-stresses are the main source of variation in the value of $\mathrm{SI}_{\mathrm{N}}$. However, for larger specimen sizes, when the influence of the T-stresses is nullified from the existence of the void, as at the neutral point, stress intensity seems to be exclusively controlled by the significance of the $A_{1 / 2}$ - non-singular stresses with a hyperbolic relationship, independent of both specimen and void size (Figure 6 ). More specifically, when the $A_{1 / 2}$-stresses become the dominant term (for small K-dominant zone size), the value of $\mathrm{SI}_{\mathrm{N}}$ is the highest. This is more closely investigated by viewing the material macroscopically.

\subsection{Macroscale: Homogenized material}

The topology-dependent stress variation can be circumvented by introducing an average normalised stress intensity $\overline{S I}_{N}$ per model, which results from averaging the local $\mathrm{SI}_{\mathrm{N}}$ values derived for relative crack tip locations $s x=0-0.75$ ( $s x=1$ is not considered due to periodicity of the solution). Thus, we can consider each specimen to be a homogenized medium of given porosity and size, without focusing on the specifics of its mesostructure.

The estimated average values are plotted in Figure 7a. Previous research on the fracture properties of porous materials has mainly focused on infinite media, assuming that any specimens cut from the same brittle material, characterized by the same porosity and microstructural features, will in fact exhibit the same fracture behaviour [34-36]. However, this study suggests that, for constant porosity, smaller specimens will appear tougher than their larger counterparts since they exhibit lower stress intensities for the same loading. In fact, 1-row specimens have significantly different behaviour than larger specimen, which seem to converge asymptotically to an approximately constant value above four rows of unit cells. This size effect becomes more pronounced with increasing porosity (Figure 7a).

Figure 7a also shows that stress at the crack tip is proportional to the porosity of the specimen, with all porous materials exhibiting higher stress intensity at the crack tip than the homogeneous case. This implies that a lower load than anticipated for the homogeneous case is required to propagate the crack in the porous materials, which complies with theory and experiments [1]. The averaged stress for the model with the non-symmetric pattern around the crack (inset of Figure 5c) is also depicted (dark blue, dashed line in Figure 7a and b). Even though its mesoscopic behaviour is distinctively different to its symmetric counterpart, macroscopically their behaviour is almost identical.

LEFM predicts that when the size of the K-dominant zone is large enough to fully engulf the fracture process zone, the value of the stress at the crack tip is purely dependent on the value of the SIF and should remain constant. For porous materials, the size of the K-dominant zone is largely affected by the existence of discontinuities in the material. The difference between the size of the K-dominant zone in the homogeneous case and the porous specimen is 
monotonically rising with increasing size (Figure 7b). Even a porosity of $5 \%$ can shrink the size of the K-dominance zone to almost half its original size for just 2 rows of unit cells. In that case it is not immediately evident that the FPZ is in all cases smaller than the K-dominant zone and thus it cannot be concluded that the $A_{1 / 2}$-stresses can be safely neglected.

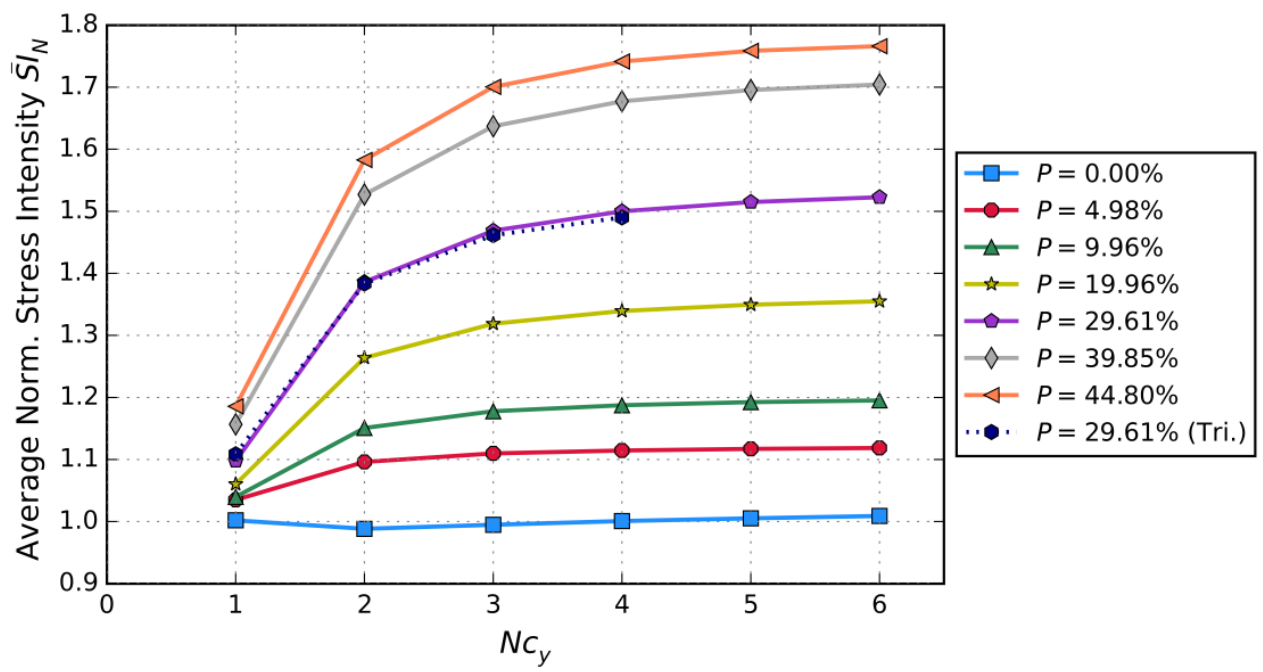

a)

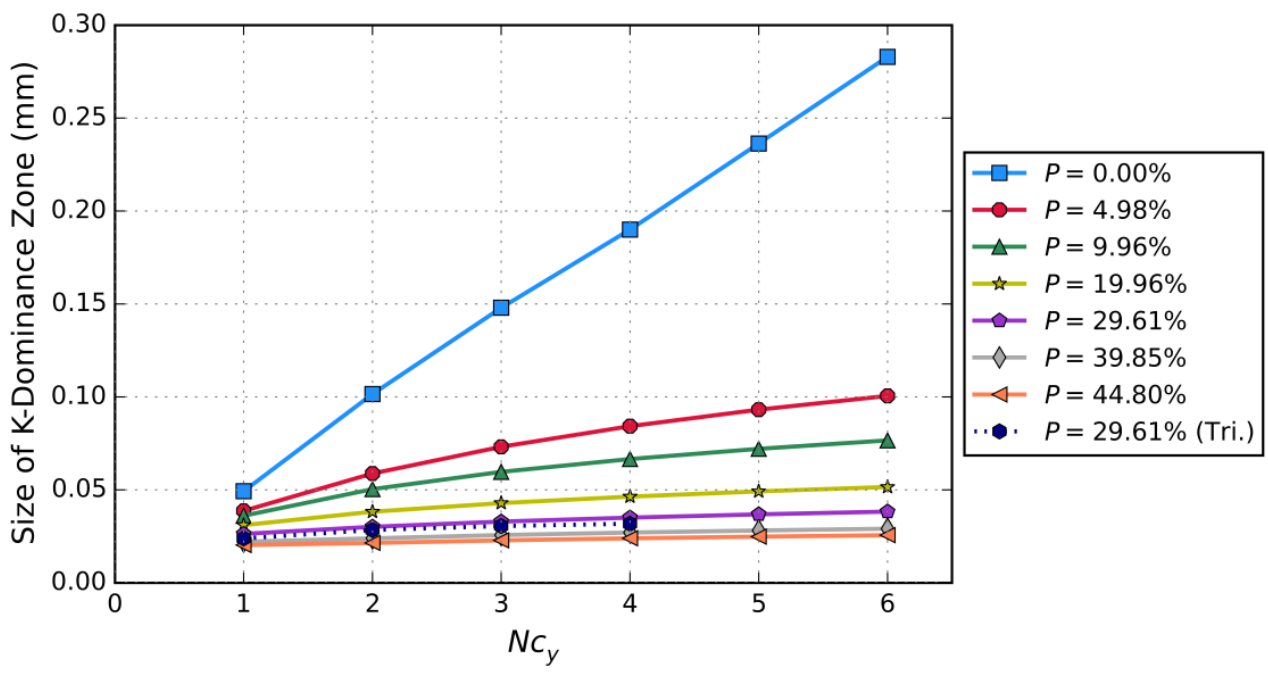

b)

Figure 7. Size effect in fracture of porous materials. a) Smaller specimen exhibit lower stresses for a given load, implying a tougher structure. The larger the porosity the more prominent the size effect. b) Dependence of size Kdominant zone with thickness of specimen.

Indeed, plotting the average normalised stress intensity at the crack tip as a function of the averaged normalized amplitude of $A_{1 / 2}$-stresses, $\tilde{C}$, we see an almost linear relationship as $\tilde{C}$ approaches zero (Figure 8a). With the exception of the smallest specimen, the magnitude of the slope of this line increases with decreasing specimen size. As $\tilde{C}$ reaches the value of the homogenous case, all series converge to the stress value for this case. It is suggested that the average normalised stress intensity at the crack tip and the normalized amplitude of the $A_{1 / 2}$ stresses $\tilde{C}$ are related by: 


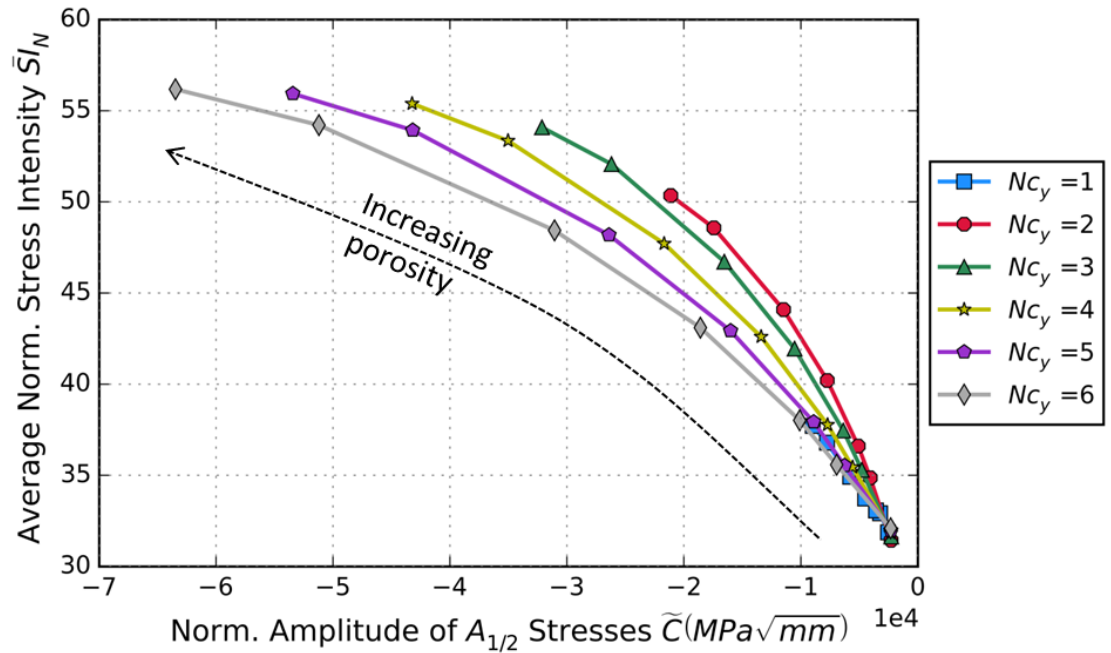

a)

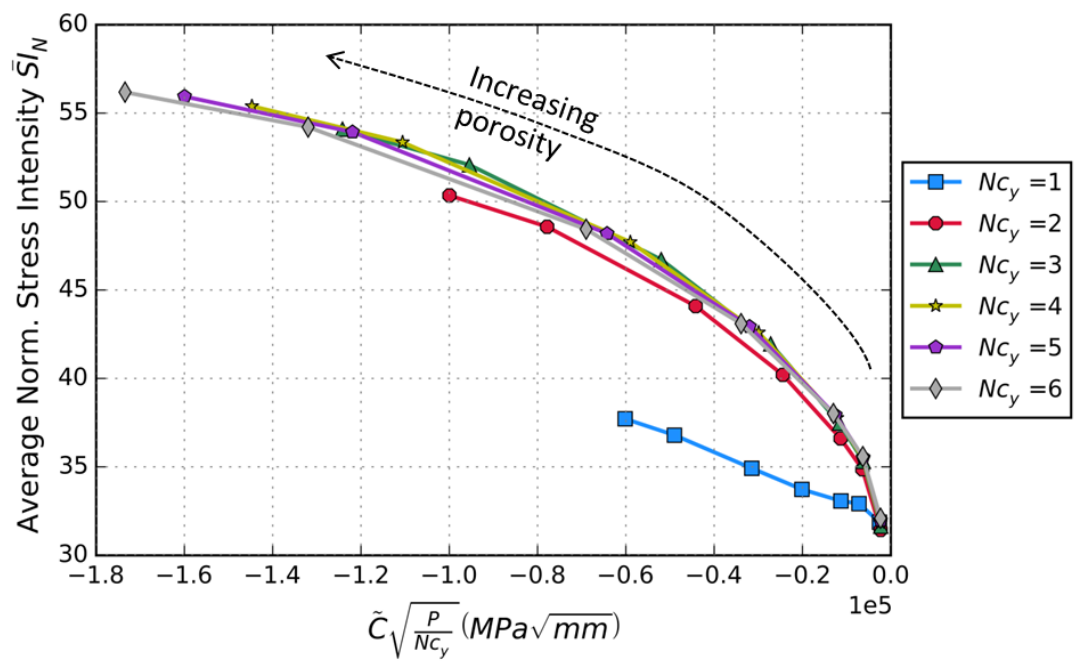

b)

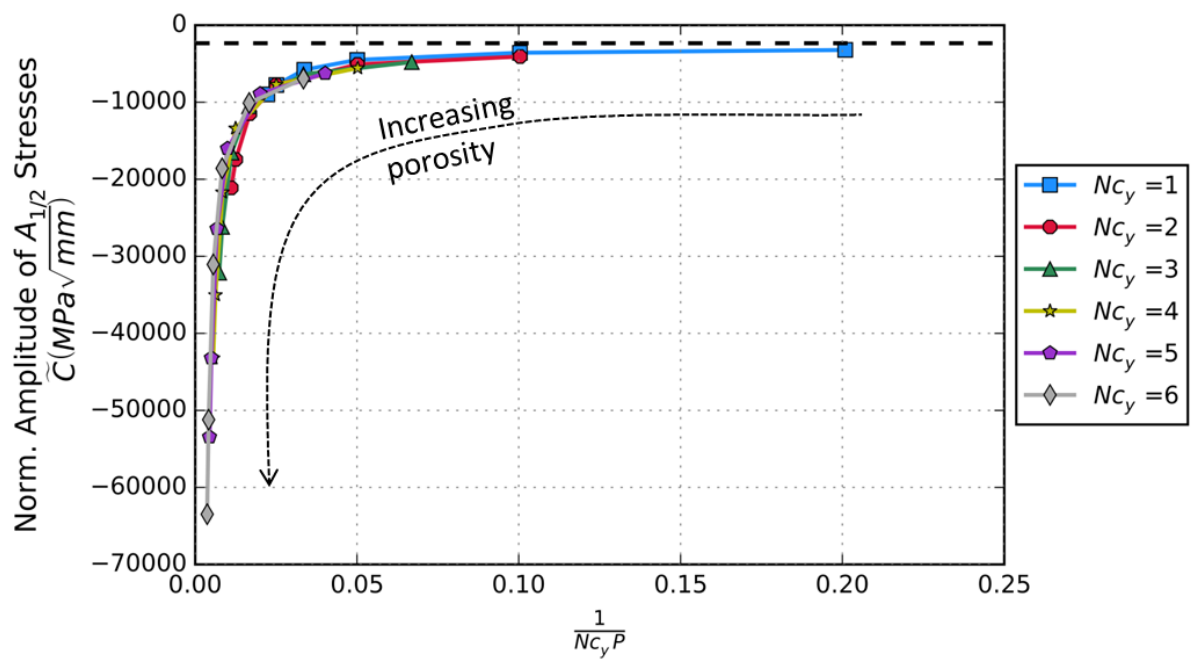

Figure 8. a) Average normalised stress intensity $\overline{S I}_{N}$ variation with averaged normalised amplitude of $A_{1 / 2}$-stresses $\widetilde{C}$, b) Inclusion of correction factor $\sqrt{\frac{N c_{y}}{P}}$ in graph a), c) Dependence of normalised amplitude of $A_{1 / 2}$-stresses $\widetilde{C}$ with number of cells in y-direction, Ncy, and specimen porosity, P. The horizontal dashed line represents the value of $\widetilde{C}$ for the homogeneous case. 


$$
\bar{\sigma}_{y y, N}=f\left(\tilde{C} \sqrt{\frac{P}{N c_{y}}}\right)
$$

where $\mathrm{Nc}_{\mathrm{y}}=$ Number of cells in $\mathrm{y}$-direction (-)

$$
\mathrm{P}=\text { Specimen porosity (-) }
$$

The data of Figure $8 \mathrm{a}$ have been re-plotted in Figure $8 \mathrm{~b}$ taking into account equation (8). It is now apparent that specimens with just one row of unit cells (blue curve in Figure $8 \mathrm{~b}$ ) exhibit a behaviour inconsistent with their larger counterparts, which is attributed to the lack of isotropy.

The averaged normalized amplitude of $A_{1 / 2}$-stress $\tilde{C}$ seems to be closely related to the geometrical features of the specimen (Figure $8 \mathrm{c}$ ). As porosity and specimen size decrease, $\tilde{C}$ tends asymptotically to the value of the homogeneous case, which is the dotted line in Figure $8 \mathrm{c}$, used as a reference. This indicates that for the given loads, $\tilde{C}$ is varied as:

$$
\tilde{C}=f\left(\frac{1}{N c_{y} P}\right)
$$

Dividing the dimensional factor found in (9) with the one found in (8) we get the geometrical dependency coefficient:

$$
D_{c}=\sqrt{\frac{1}{N c_{y} P^{3}}}
$$

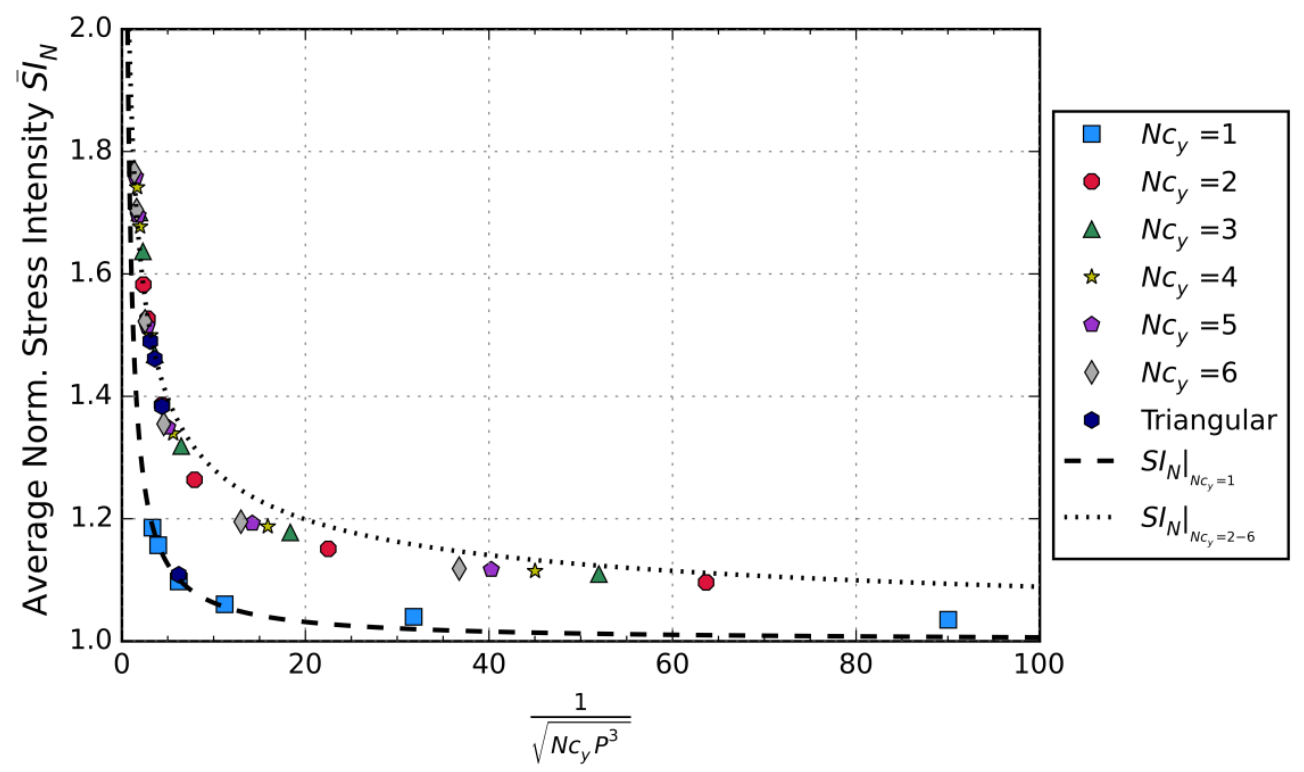

Figure 9. Correlation of scaling factor with the geometrical dependency coefficient. The fitted equations $\mathbf{1 1}$ is also plotted on the graph.

The average normalised stress intensity at the crack tip $\overline{S I}_{N}$ can thus be accurately plotted as a function of the dependency coefficient (Figure 9). Through parameter fitting we estimate the following relation: 


$$
\left.\overline{S I}_{N}\right|_{N_{c y=i}}= \begin{cases}\frac{0.89}{D_{c} \sqrt{2}}+1 & \text {, if } i=1 \\ \frac{0.89}{\sqrt{D_{c}}}+1 & \text {, if } i>1\end{cases}
$$

, where the standard error of estimate $s_{\text {est }}$ for specimen with 1 row of unit cells is $s_{\text {est }}=0.014$, while for larger specimen $(i>1) s_{\text {est }}=0.035$.

The exact value of the average stress intensity at the crack tip of the porous material $\overline{S I}^{P}$ for a given load can then be estimated from equation (4) as:

$$
\overline{S I}^{P}=\left.\overline{S I}_{N}\right|_{N_{c y=i}} * \overline{S I}^{H}
$$

It is interesting to note that even the values of the model with the triangular non-symmetric void arrangement about the crack also comply with this formula. However, in order to make safe assumptions about the applicability of the formula to materials of different topologies and lesser periodicity, a thorough parametric study needs to be run.

\section{Conclusions}

The present study investigates the influence of specimen size and porosity on the stress at the crack tip both mesoscopically (at the pore level) and macroscopically (at the homogenized material level), before the critical stress intensity value. The results indicate that it is inadequate to refer to one unique stress intensity for porous materials, as the inclusion of only singular stresses would suggest. The presence of the pores, as well as the size of the specimen, have a direct impact on the amplitude of non-singular stresses on each model, which is subsequently reflected on the stress intensity at the crack tip of the materials studied. Furthermore, when the size of the specimen is reduced to just one row of unit cells, the fracture behaviour exhibited is not consistent with the behaviour of larger specimen.

In summary, the following conclusions can be drawn:

1. The crack tip stress-field at each specific location relative to the void is influenced by a combination of the $\mathrm{x}$ - and $\mathrm{y}$-direction non-singular stresses ( $\mathrm{T}$ - and $\mathrm{A}_{1 / 2^{-}}$stresses respectively). For each model the stress follows an oscillating fluctuation whose mean value is related to the normalized amplitude of $A_{1 / 2}$-stresses, $C$. The amplitude of the oscillation is dependent on the amplification of the T-stress distribution, which is directly influenced by the specific topology of the voids in the near-tip field. When the size is reduced to just one row of unit cells however the effect of the $A_{1 / 2}$-stresses diminishes, while the T-effect is much more prominent.

2. Looking at the material macroscopically we observe a prominent size effect. As the specimen size increases, the material may appear to become less tough, until it converges to a specific value, assumed to be that of the infinite medium. As expected, a weakening effect is also 
exhibited with increasing porosity. Both behaviours are closely related to the amplification of the $A_{1 / 2}$-stresses, which become significant when compared to singular stresses, rendering the consideration of higher-order terms necessary in predicting the fracture behaviour of heterogeneous specimens.

3. An empirical relation has been derived to predict the average stress intensity at the crack tip of porous materials with low to medium porosity. The model was created based on FEA data obtained for DCB specimens where the porosity was introduced in the form of circular unit cells in a periodic array.

The above study raises interesting questions on whether the $A_{1 / 2^{-}}$non-singular stresses are equally significant in non-symmetric loading modes and geometries.

\section{References}

[1] Gibson LJ, Ashby MF. Cellular Solids. vol. 2. Cambridge: Cambridge University Press; 1997. doi:10.1017/CBO9781139878326.

[2] Lau APS, Chen G-F. Porous materials : processing and applications. Elsevier Science; 2014.

[3] Wei W, Xia Y. Geometrical, fractal and hydraulic properties of fractured reservoirs: A mini-review. Ausasia Sci Technol Press Adv Geo-Energ Res Adv Geo-Energ Res 2017;1:31-8. doi:10.26804/ager.2017.01.03.

[4] Berkowitz B. Characterizing flow and transport in fractured geological media: A review. Adv Water Resour 2002;25:861-84. doi:10.1016/S0309-1708(02)00042-8.

[5] Kabir ME, Saha MC, Jeelani S. Tensile and fracture behavior of polymer foams. Mater Sci Eng A 2006;429:225-35. doi:10.1016/j.msea.2006.05.133.

[6] Linul E, Marsavina L, Sadowski T, Knec M. Size Effect on Fracture Toughness of Rigid Polyurethane Foams. Solid State Phenom 2012;188:205-10. doi:10.4028/www.scientific.net/SSP.188.205.

[7] Marsavina L, Linul E. Fracture toughness of polyurethane foams. Experiments versus micromechanical models. Proc 18th Eur. Conf. Fract., 2010, p. 1-8.

[8] Rice JR. Mathematical Analysis in the Mechanics of Fracture. Fract An Adv Treatise Math Fundam NY 1968;2:191-311.

[9] Anderson TL. Fracture Mechanics: Fundamentals and Applications. 2005.

[10] Larsson SG, Carlsson AJ. Influence of non-singular stress terms and specimen geometry on smallscale yielding at crack tips in elastic-plastic materials. J Mech Phys Solids 1973;21:263-77. doi:10.1016/0022-5096(73)90024-0.

[11] Rice JR. Limitations to the small scale yielding approximation for crack tip plasticity. J Mech Phys Solids 1974;22:17-26. doi:10.1016/0022-5096(74)90010-6.

[12] Gardeazabal D, He Z, Kotousov A. On influence of non-singular stress states on brittle fracture. Int J Fract 2014;185:201-8. doi:10.1007/s10704-013-9903-7.

[13] Kumar B, Chitsiriphanit S, Sun CT. Significance of K-dominance zone size and nonsingular stress field in brittle fracture. Eng Fract Mech 2011;78:2042-51. doi:10.1016/j.engfracmech.2011.03.015.

[14] Chao YJ, Liu S, Broviak BJ. Brittle fracture: Variation of fracture toughness with constraint and crack curving under mode I conditions. Exp Mech 2001;41:232-41. doi:10.1007/BF02323139.

[15] Kravchenko OG, Kravchenko SG, Sun C-T. Thickness dependence of mode I interlaminar fracture toughness in a carbon fiber thermosetting composite 2017. doi:10.1016/j.compstruct.2016.10.088.

[16] Smith DJ, Ayatollahi MR, Pavier MJ. On the consequences of T-stress in elastic brittle fracture. Proc R Soc A Math Phys Eng Sci 2006;462:2415-37. doi:10.1098/rspa.2005.1639.

[17] Han JJ, Chen YH. T-effect for the interaction problem of an interface macrocrack with a near-tip microvoid. Int J Fract 2000;102:205-22. doi:10.1023/A:1007533120851. 
[18] Thomas S., Mhaiskar M., Sethuraman R. Stress intensity factors for circular hole and inclusion using finite element alternating method. Theor Appl Fract Mech 2000;33:73-81.

doi:10.1016/S0167-8442(00)00002-1.

[19] Zhou C, Li Z. The effect of T-stress on crack-inclusion interaction under mode I loading. Mech Res Commun 2007;34:283-8. doi:10.1016/j.mechrescom.2006.10.001.

[20] Bazant ZP, Zhou Y, Zi G, Daniel IM. Size effect and asymptotic matching analysis of fracture of closed-cell polymeric foam. Int J Solids Struct 2003;40:7197-217. doi:10.1016/S00207683(03)00362-7.

[21] Bazant ZP, Daniel IM, Zhengzhi L. Size Effect and Fracture Characteristics of Composite Laminates. J Eng Mater Technol 1996;118:317-24.

[22] Bazant ZP, Zhou Y, Novák D, Daniel IM. Size effect in fracture of sandwich structure components: Foam and laminate. Am Soc Mech Eng Appl Mech Div AMD 2001;248:19-30.

[23] Bazant ZP. Scaling laws in mechanics of failure. J Eng Mech 1993.

[24] Bazant ZP, Xi Y, Reid SG. Statistical size effect in quasi-brittle structures: I. Is Weibull theory applicable? J Eng Mech 1991;117:2609-22.

[25] Bazant ZP. Concrete fracture models : testing and practice. Eng Fract Mech 2002;69:165-205.

[26] Wheel MA. A control volume-based finite element method for plane micropolar elasticity. Int J Numer Methods Eng 2008:992-1006. doi:10.1002/nme.2293.

[27] Wheel MA, Frame JC, Riches PE. Is smaller always stiffer ? On size effects in supposedly generalised continua. Int J Solids Struct 2015;67-68:84-92. doi:10.1016/j.ijsolstr.2015.03.026.

[28] Dunn MA, Wheel MA. Computational Analysis of the Size Effects Displayed in Beams with Lattice Microstructures, 2016, p. 129-43. doi:10.1007/978-3-319-31721-2_6.

[29] Wheel MA, Frame JC, Riches PE. Paradoxical Size Effects in Composite Laminates and Other Heterogeneous Materials, 2016, p. 443-57. doi:10.1007/978-3-319-31721-2_21.

[30] Williams ML. On the Stress Distribution at the Base of a Stationary Crack. J Appl Mech 1957;24:109-14. doi:10.1115/1.3640470.

[31] Zehnder AT. Linear Elastic Stress Analysis of 2D Cracks, Springer, Dordrecht; 2012, p. 7-32. doi:10.1007/978-94-007-2595-9_2.

[32] Morioka Y. A simple method for determining stress intensity factors for a crack in bi-material interface. Theses Diss Available from ProQuest 2010.

[33] Sun CT, Qian H. Brittle fracture beyond the stress intensity factor. J Mech Mater Struct 2009;4:743-53.

[34] Leguillon D, Piat R. Fracture of porous materials - Influence of the pore size. Eng Fract Mech 2007:1840-53. doi:10.1016/j.engfracmech.2006.12.002.

[35] Vandeperre LJ, Wang J, Clegg + WJ. Effects of porosity on the measured fracture energy of brittle materials. Philos Mag 2004;84:3689-704. doi:10.1080/14786430412331293522.

[36] Ryvkin M, Hadar O. Employing of the discrete Fourier transform for evaluation of crack-tip field in periodic materials. Int J Eng Sci 2015;86:10-9. doi:10.1016/j.ijengsci.2014.10.001. 